\title{
Space weathering of asteroidal surfaces
}

\section{Influence on the UV-Vis spectra ${ }^{\star}$}

\author{
Z. Kaňuchová ${ }^{1,2}$, G. A. Baratta ${ }^{1}$, M. Garozzo ${ }^{1}$, and G. Strazzulla ${ }^{1}$ \\ 1 INAF-Osservatorio Astrofisico di Catania, via S. Sofia 78, 95123 Catania, Italy \\ e-mail: zuzana@oact.inaf.it \\ 2 Astronomical Institute of Slovak Academy of Sciences, 05960 Tatranska Lomnica, Slovakia
}

Received 14 January 2010 / Accepted 27 April 2010

ABSTRACT

\begin{abstract}
Context. The surfaces of airless bodies in the Solar System are continuously altered by the bombardment of micrometeoroids and irradiation by solar wind, flares, and cosmic particles. Major effects of this process - space weathering - are darkening and "reddening" of the spectra of surface materials, as well as a "degrading" of absorption features.

Aims. We studied the changes induced by energetic ion irradiation in the ultraviolet-visual-near-infrared (UV-Vis-NIR) $(0.2-0.98 \mu \mathrm{m})$ reflectance spectra of targets selected to mimic the surfaces of airless bodies in the inner Solar System. Our chosen targets are olivine pellets, pure or covered by an organic polymer (polystyrene), which is transparent before irradiation. Polystyrene is used as a template for organic matter of low volatility that can be present on asteroidal surfaces. Moreover we measured the changes induced by ion irradiation in the absorption coefficient of the polymer. The purpose was to have a tool to better compare laboratory with observed spectra and distinguish between planetary objects with pure silicate surfaces and those whose surface is covered by organic matter exposed to cosmic ion bombardment.

Methods. The samples were irradiated in vacuum, at room temperature, with $200 \mathrm{keV}$ protons or $200-400 \mathrm{keV}$ argon ions. Before, during, and after irradiation diffuse reflectance spectra were acquired. Polystyrene films were also deposited on quartz substrates and irradiated while transmittance spectra were recorded.

Results. We measured the variations of the absorption coefficient of polystyrene as a function of ion fluence. We showed that after ion irradiation the diffuse reflectance spectra of the samples covered by organics exhibit a much more significant variation than those of pure silicates. The spectra of targets made of olivine plus polystyrene can be fitted by using the measured absorption coefficient of polystyrene.

Conclusions. The results obtained for pure olivine extend to the UV the spectral range of previous experiments. The data concerning the absorption coefficient of polystyrene are available on our web site (http://web.ct.astro.it/weblab/dbindex.html) and can be used to compare laboratory with astronomical spectra. This will possibly allow us to obtain information about organic matter on the surface of a given object, as well as on the relevance of the exposure to cosmic ions (space weathering).
\end{abstract}

Key words. minor planets, asteroids: general - methods: laboratory - techniques: spectroscopic

\section{Introduction}

It is well known that the surfaces of airless bodies in the Solar System are continuously altered by the bombardment of micrometeoroids and irradiation by solar wind, flares, and cosmic particles. Major effects of this process - space weathering - are darkening and "reddening" (spectral reflectance increases with wavelength) of the spectra of surface materials, as well as "degrading" of absorption features (Chapman 1996; Pieters et al. 1993). Up to now most of the laboratory simulations of space weathering, in particular ion irradiation, have been conducted by looking at changes in the spectra of relevant materials at visible and near-IR (NIR) wavelengths $(0.5-2.5 \mu \mathrm{m})$.

Investigated materials include carbon-based species such as frozen hydrocarbons, present on the outer Solar System objects and interstellar dust grains (Brunetto et al. 2006a), red bitumens (asphaltite and kerite, materials which are the most similar to the less evolved organic matter of trans-neptunian

* Data table is only available in electronic form at the CDS via anonymous ftp to cdsarc.u-strasbg.fr (130.79.128.5) or via http://cdsweb.u-strasbg.fr/cgi-bin/qcat?]/A+A/517/A60 objects (TNOs) and comets) (Moroz et al. 2004), and materials typical for main-belt asteroids such as different kinds of silicates (Brunetto \& Strazzulla 2005; Brunetto et al. 2006b; Sasaki et al. 2001; Strazzulla et al. 2005; Loeffler et al. 2009, 2008). Up to now, the effects of space weathering have not yet been studied on organic materials of low volatility. Such materials macromolecules or molecular solids - could be very abundant on small objects in the outer Solar System, where they could be accreted directly from the nebular material at the beginning of the Solar System or they could be produced by energetic processing of simple ices (e.g. Palumbo et al. 2008). The same molecules or their space weathered daughters could be present on closer objects such as asteroids or even Mars satellites (Phobos and Deimos, e.g. Shkuratov et al. 1987).

According to the work of Hendrix \& Vilas (2006), the space weathering effects could be more evident in the UV range than in the visible and NIR ones. It was found a correlation between the visible-near-IR (VNIR, 550-1640 nm) and near-UV (NUV, $300-400 \mathrm{~nm}$ ) spectral slopes of S-class asteroids and ordinary chondrites, which suggests that surfaces that are spectrally redder in the VNIR are also spectrally bluer in the NUV 
(Hendrix \& Vilas 2006). In the spectra of most lunar-like and asteroid-like minerals an absorption feature in NUV appears as a result of a transition between volume scattering and surface scattering. The strength of this feature depends on the presence of opaque/nonopaque materials (e.g. pyroxenes, feldspars, and olivines all exhibit a UV drop-off in the $150-450 \mathrm{~nm}$ range that is not seen in iron-bearing minerals as ilmenite and magnetite). The relative blue spectral slope in the NUV can be explained by a decrease in the strength of the UV absorption feature due to weathering-derived nanophase iron. This suggests that surfaces of different materials will manifest weathering in different ways.

The UV wavelength region is suggested to be a more sensitive indicator of weathering (and thus age), because the changes in this spectral region should be more evident than those at longer wavelengths for the same exposure to weathering processes. In some cases, the effects of space weathering in UV-visible wavelengths can include also a spectral reversal besides the bluing of spectral reflectance which means that relatively dark surfaces in the visible range are relatively bright at shorter wavelengths (Hendrix \& Vilas 2006).

In our Laboratory for Experimental Astrophysics (LASp) in Catania (Italy) we started with a new series of experiments aimed at studying the effects of ion irradiation in solid materials in a spectral range that includes the NUV $(0.2-0.98 \mu \mathrm{m})$ region.

Here we present the results obtained so far that concern ion irradiation of olivine samples and olivine samples that were prepared with a coat of polystyrene films. Olivine was used as an example of the asteroidal surface and polystyrene was chosen as a template for organic materials of low volatility, possibly present on the surfaces of some small Solar System objects. Moreover, we measured the changes induced by ion irradiation in the absorption coefficient of the polymer. The purpose was to have a tool to better compare laboratory with observed spectra and distinguish between planetary objects with pure silicate surfaces from those whose surface is contaminated by organic matter exposed to cosmic ion bombardment. Polystyrene is meant to mimic a kind of organic material (i.e. a spectrally neutral one) freshly exposed at the surface of minor objects in the Solar System.

Polystyrene (Poly(1-phenylethane-1,2-diyl), $\left.\left(\mathrm{C}_{8} \mathrm{H}_{8}\right)_{n}\right)$ is an aromatic vinyl polymer made from the aromatic monomer styrene $\left(\mathrm{C}_{6} \mathrm{H}_{5} \mathrm{CH}=\mathrm{CH}_{2}\right)$. Structurally, it is a long hydrocarbon chain, with a phenyl group attached to every other carbon atom. Polystyrene is a transparent thermoplastic substance existing in solid state at room temperature. The strong absorption of polystyrene in the UV spectral range (with the onset at $280 \mathrm{~nm}$ ) is well known (e.g. Partridge 1967).

\section{Experimental apparatus}

Iradiation experiments were carried out at room temperature inside a stainless steel vacuum chamber $\left(P<10^{-7}\right.$ mbar $)$, faced to near-UV-visual-near-IR (NUV-Vis-NIR) $(0.2-0.98 \mu \mathrm{m}) \mathrm{mini}-$ spectrometer. Depending on the type of experiment and kind of sample, the configuration for reflectance or transmittance measurement can be used. In the center of the vacuum chamber the flat round-shaped sample is held up on a finger by a metal corona ring. Samples are irradiated inside the chamber with different ions using a Danfysik (1080-200) ion implanter. Ions with an energy from 30 up to $200 \mathrm{keV}$ (400 keV for double ionization) can be obtained. The ion beam passes through a sweeping system, producing a uniform coverage over an area of about $4 \mathrm{~cm}^{2}$, thus irradiating the whole surface of the sample. The currents

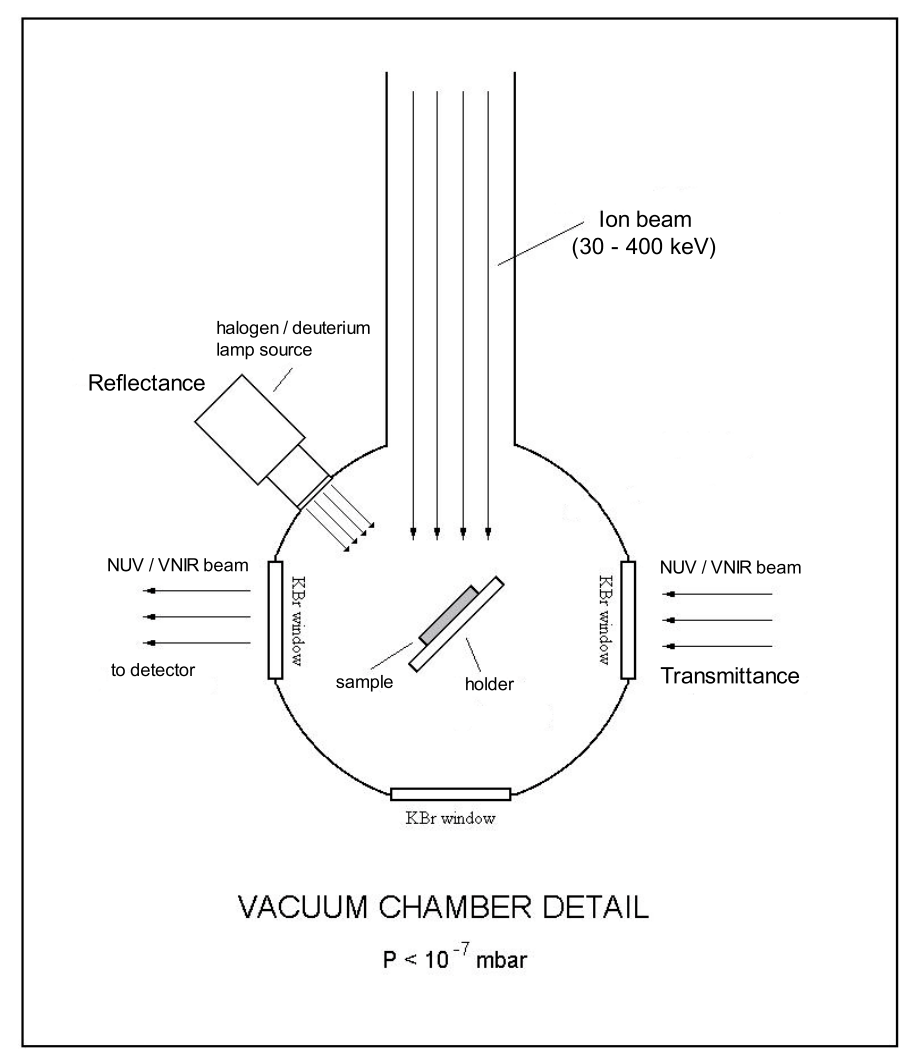

Fig. 1. Scheme of the vacuum chamber: solid samples are held on a finger. The chamber is interfaced to an ion implanter. Bi-directional reflectance spectra are acquired with a halogen/deuterium lamp source and the NUV-Vis-VNIR spectrometer.

are below $1 \mu \mathrm{m}$ to avoid macroscopic heating of the sample. The ions impinge the surface of the sample at an angle of $45^{\circ}$.

During experiments the bi-directional reflectance/transmittance spectra are achieved using NUV-VIS-NIR spectrometer with two sources (halogen and deuterium lamp), which cover the spectral range $200-980 \mathrm{~nm}$. However, the background signal of the deuterium lamp decreases rapidly below $250 \mathrm{~nm}$. For this reason we considered as relevant only the data at longer wavelengths. The lamps, with a partially overlapping spectral region around $450 \mathrm{~nm}$, were used separately, and the two parts of the achieved spectra were subsequently joined. For the reflectance measurements configuration, the scattered light is collected by the spectrometer at an angle of $45^{\circ}$ with respect to the incident radiation that is normal to the sample surface. Because the ion beam forms an angle of $45^{\circ}$ with the target plane and of $90^{\circ}$ with the collected NUV/VNIR radiation, spectra can be obtained before, during and after the irradiation without tilting the sample. For the transmittance measurements, the beam of incident radiation makes the angle of $45^{\circ}$ with the surface of the studied sample. A schematic view of the experimental apparatus is shown in Fig. 1.

To measure the thickness of the polystyrene layer deposited on the olivine pellets, ex-situ hemispherical reflectance spectra of nonirradiated samples and transmittance spectra of polystyrene film in the range $0.25-2.5 \mu \mathrm{m}$ were acquired with the UV-Vis-NIR Spectrophotometer Perkin-Lambda 19. Type of spectrometer and configuration of measurement is specified below for every performed experiment. 


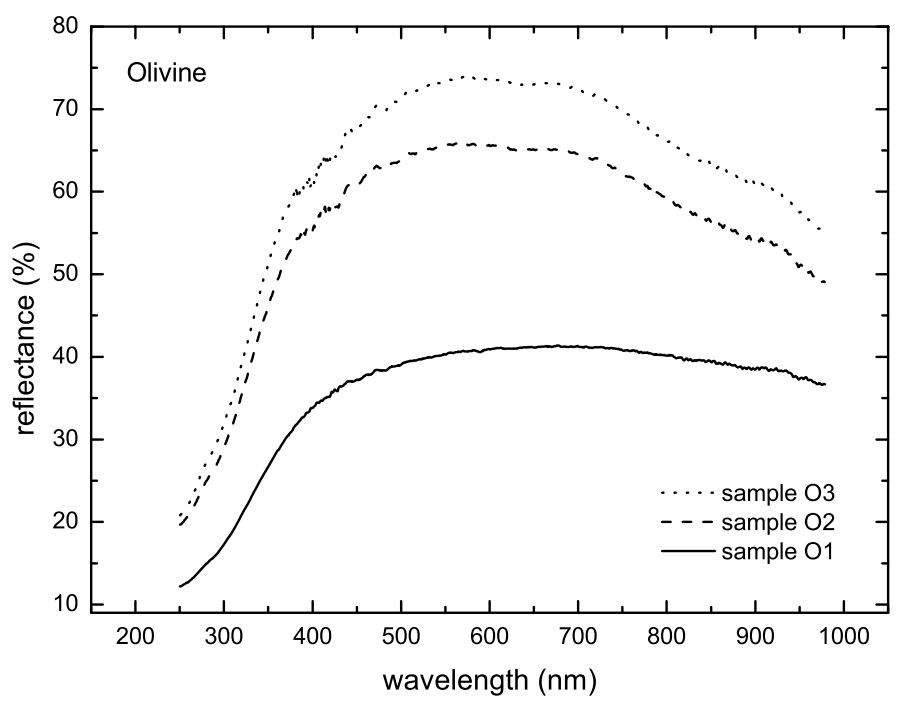

Fig. 2. Reflectance spectra of three olivine pellets in UV/Vis/NIR spectral region.

\section{Experiments}

Three types of experiments were performed.

\subsection{Irradiation of olivine}

We prepared three olivine samples (labeled O1-O3), which consist of pressed olivine $\left((\mathrm{Mg}, \mathrm{Fe})_{2} \mathrm{SiO}_{4}\right)$ powder (milled olivine rocks) deposited on previously pressed $\mathrm{KBr}$ pellets. The reflectance spectra of unirradiated olivine samples show strong and broad features slightly longward of $1 \mu \mathrm{m}$, which is due to ferrous iron $\mathrm{Fe}^{2+}$ in olivine. Actually, the feature is a blend of three unresolved absorption bands due to the excitation of an electron on $\mathrm{Fe}^{2+}$ surrounded by six oxygen ions (Brunetto \& Strazzulla 2005; Hapke 2001, and references therein).

The wing of the UV exciton/valence-conduction band system below $200 \mathrm{~nm}$ causes the reflectance of silicate powders to decrease with decreasing wavelength (Hapke 2001). The decrease in the NUV is also clearly seen in the spectra of the bulk silicate samples (Brunetto \& Strazzulla 2005) and in the spectra of the samples studied here (Fig. 2). Differences in reflectance of the three samples are attributed to differences in the surface roughness (particle size distribution of olivine powder used). Two samples were irradiated with $400 \mathrm{keV}$ and $200 \mathrm{keV}$ Ar ions; the irradiation of the third one was done with $200 \mathrm{keV}$ protons.

When ion irradiation experiments are performed to simulate solar wind and cosmic ion irradiation, the corresponding type of ion can easily be obtained in a laboratory, though it is more difficult to reproduce the energy distribution of these ions. Therefore, the laboratory results can be used for astrophysical application only when we find out which is the physical mechanism that is causing the observed effects, i.e. when we can extrapolate the laboratory data to a wider range of ion masses and energies. $400 \mathrm{keV}$ Argon ions and $200 \mathrm{keV}$ protons have been chosen as representative of two different kinds of possible solar ions that deposit their energy trough mainly inelastic interactions ( $200 \mathrm{keV}$ protons) and both inelastic and elastic interactions $\left(400 \mathrm{keV} \mathrm{Ar}^{++}\right)$.

The variation of the color of the samples induced by different ions with different energies can be quantified by measuring the spectral slopes in different spectral regions. To achieve the comparable quantity, the slopes had to be computed for the normalized spectra. The spectral slopes in units of reflectance $\mathrm{nm}^{-1}$

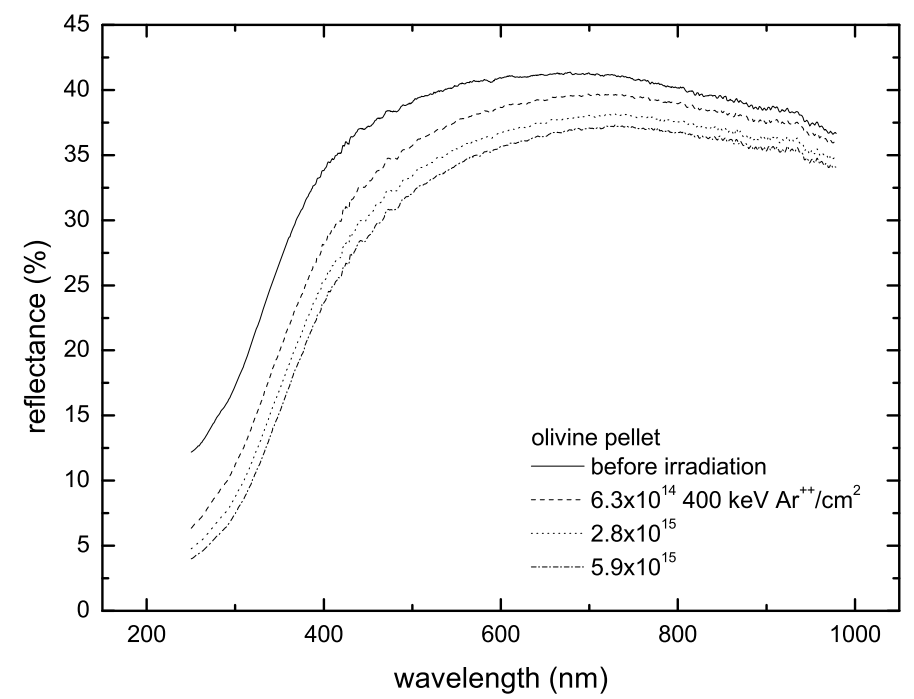

Fig. 3. Reflectance spectra of olivine pellet $\mathrm{O} 1$ measured "in situ" during the ion irradiation. Total fluence values in units of ions $/ \mathrm{cm}^{2}$ are given.

were computed by fitting a straight line to the reflectance data scaled to unity at $\lambda=550 \mathrm{~nm}$ (method of linear regression) in the studied wavelength range. The spectral slopes at near UV $(300-400 \mathrm{~nm})$ of all samples at increasing ion fluences during irradiation were determined. As an example, the reflectance spectra of one pellet are shown in Fig. 3. The change of the NUV spectral slope of the samples with increasing ion fluence can be seen.

The spectral slopes of all the samples before and after irradiation at near UV (300-400 nm) wavelengths were determined and will be shown and discussed later in this paper (Fig. 8).

\subsection{Experiments with polystyrene}

We prepared five different samples of polystyrene (labeled P1P5) deposited onto quartz windows. A solution of polystyrene in chloroform was prepared and drops deposited onto a spinning quartz windows to get a uniform film. With solutions of different concentration we got polystyrene layers of different thickness. The thickness of the deposited layers was determined indirectly by comparing the intensity of several IR absorption bands (in an optical depth scale) with those of a standard film (Perkin Elmer) whose thickness was known $(50 \mu \mathrm{m})$ (measurement was done using Bruker Vertex 70 spectrometer). In detail it was determined by the measured optical depth

$\tau=\alpha s$

(where $\alpha$ is the absorption coefficient and $s$ the thickness of the sample) of a few non-saturated absorption bands visible in the IR spectral region.

Ion irradiation of two samples of thickness $s=(5.71 \pm 0.6) \times$ $10^{-2} \mu \mathrm{m}$ (sample P3) and $s=(2.55 \pm 0.2) \times 10^{-2} \mu \mathrm{m}$ ( ple P1) was carried out. $200 \mathrm{keV}$ (total fluence $1.6 \times 10^{16} \mathrm{~cm}^{-2}$ ) protons and $400 \mathrm{keV} \mathrm{Ar}^{++}\left(5.2 \times 10^{15} \mathrm{~cm}^{-2}\right)$ ions were used. On the basis of transmittance measurements the relative absorption coefficient $\alpha(\lambda)=\tau(\lambda) / s\left[\mathrm{~cm}^{-1}\right]$ was determined (Fig. 4) as a function of wavelength for the different ion fluences. By "relative absorption coefficient $\alpha$ " we mean the coefficient expressing the amount of radiation transmitted by irradiated material relatively to the radiation transmitted by the unirradiated sample; as 

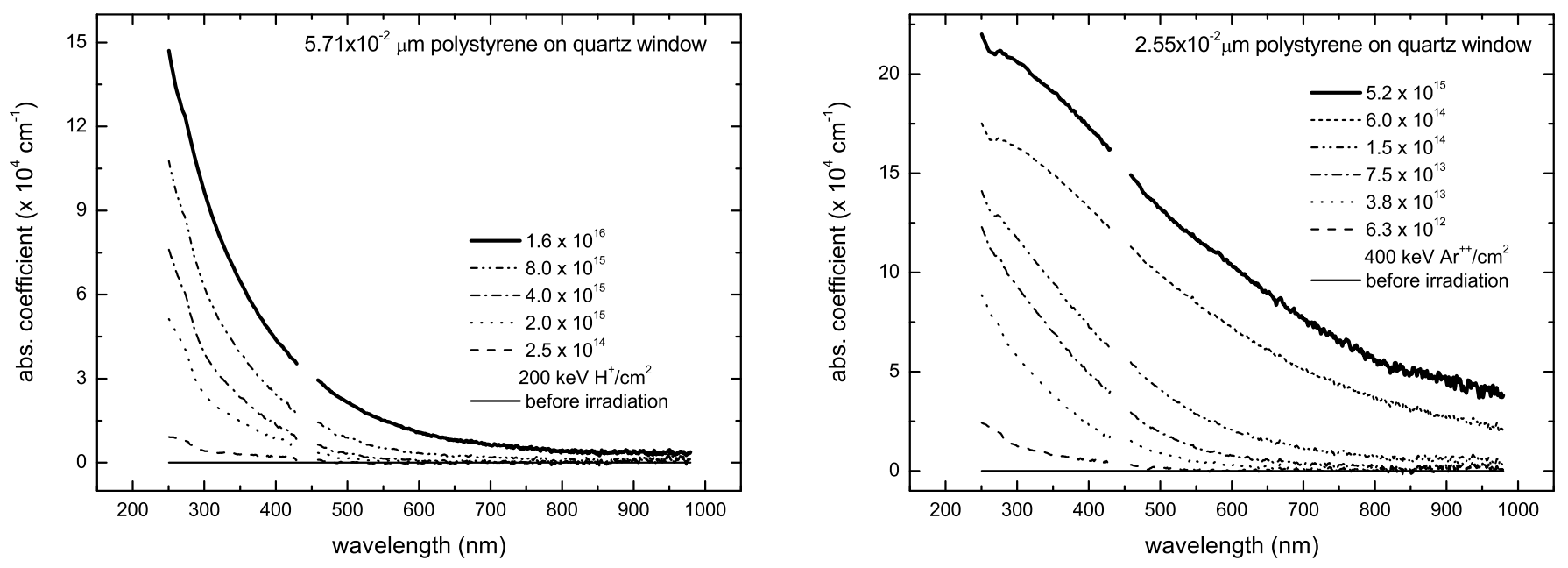

Fig. 4. Relative absorption coefficient of polystyrene. (The coefficient for the non-irradiated polystyrene is considered to be equal to zero in the whole wavelength range.)

it appears in the formula: $I_{r}=I_{0} \mathrm{e}^{-K s}$, where $I_{r}$ is the transmittance of irradiated and $I_{0}$ of unirradiated polystyrene layer of thickness $s$. For the determination of the absorption coefficient, the "effective" thickness of the polystyrene layer was used; i.e. the optical path-length throw of the polystyrene layer. A rough estimate of the optical path at oblique incidence can be obtained by the relation

$$
s=\frac{d}{\sqrt{1-\frac{\sin ^{2} i}{n^{2}}}},
$$

where $d$ is the geometrical thickness, $i$ is the incidence angle ( $45^{\circ}$ in our case) and $n$ the refractive index.

This equation does not take into account the multiple reflections occurring at the vacuum-film and film-substrate interfaces that further increase the optical path at oblique incidence. In order to consider this effect, we evaluated the effective optical path, once and for all, by comparing the in situ transmittance spectra at the highest (last) fluence with the transmittance spectra of the same sample acquired ex-situ at near-normal incidence angle. The effective optical path was that giving the best match between the in situ and ex situ absorption coefficients in the common wavelength range. The effective optical path derived in this way was 1.15 times higher than that provided by Eq. (2).

A further experimental issue that must be taken into account in the derivation of the absorption coefficient is the continuum level stability of the in situ spectrometer. In particular, small variation in the light sources intensity are expected during the several hours needed to complete a run on a sample. Although the continuum variation is expected to be small, merely within a few percent, the absorption coefficient at longer wavelengths and at lower fluences is also relatively small and could be affected by that instability. In particular, obtained optical gaps based on this absorption coefficient could be heavily affected. In order to take these possible variations into account, we allowed the continuum level to change by considering a floating multiplying factor of the whole transmittance spectra. For each irradiation step, the corrective multiplying factor was determined, in an iterative procedure, by using the feedback provided by the reflectance spectra of much thicker irradiated polystyrene films deposited over olivine (see next section). All corrective multiplying factors obtained were well within $7 \%$.

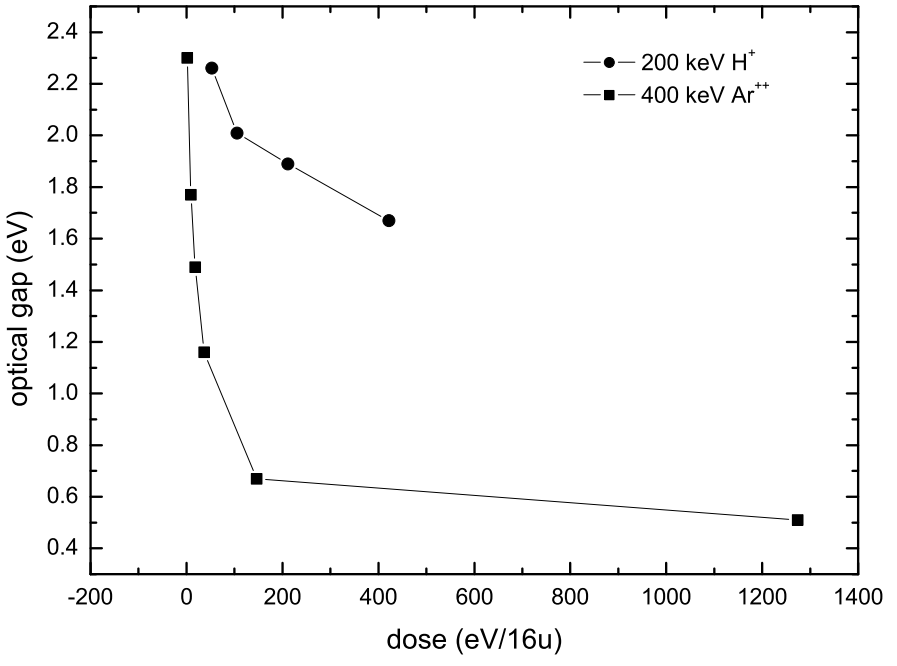

Fig. 5. Changes in the optical gap of polystyrene as a function of dose.

The absorption spectra of non-irradiated polystyrene is characterized by a broad peak with a maximum at $\lambda=262 \mathrm{~nm}$ and, according to Wittmershaus et al. (2002), also by several sharp features at 270,256 and $250 \mathrm{~nm}$. Upon irradiation, the originally transparent polystyrene samples are progressively converted into a material that already at low doses absorbs in the entire investigated wavelength range, not only in the UV. Absorption in the UV edge is more deep after irradiation, as can be seen in Fig. 4.

With Tauc's relation $(\alpha E)^{1 / 2} \propto E-E_{\mathrm{g}}$ (Tauc et al. 1966), where $E$ is the photon energy and $E_{\mathrm{g}}$ is the optical energy gap, the $E_{\mathrm{g}}$ was determined. The polystyrene film was converted into an hydrogenated amorphous carbon with a decreasing optical gap during the irradiation (Fig. 5) due to the growth of the clusters with a local graphite-like structure (see Compagnini et al. 1990; Zhu et al. 2000). The optical gap of polystyrene at the fluence $5.2 \times 10^{15} 400 \mathrm{keV} \mathrm{Ar}{ }^{++}$ions $/ \mathrm{cm}^{2}$ is $0.5 \mathrm{eV}$. The same value for the optical gap is given by Curró et al. (1995) for the polystyrene irradiated at comparable dose. According to Curró et al. (1995) the irradiated polystyrene at that stage should have $\mathrm{sp}^{2} \mathrm{C}$ atomic fraction of $65.4 \%$ and $\mathrm{H}$ to $\mathrm{C}$ ratio equal to $15 \%$.

We computed the total energy absorbed (dose) by polystyrene during the irradiation. The stopping power (energy lost by incoming ions for unit path length) for ions used in both 
Table 1. Energy absorbed per unit mass of the polystyrene samples exposed to ion irradiation.

\begin{tabular}{cccccccc}
\hline \hline \multicolumn{2}{c}{$2.55 \times 10^{-2} \mu \mathrm{m} ; 400 \mathrm{keV} \mathrm{Ar}$} & \multicolumn{5}{c}{$5.71 \times 10^{-2} \mu \mathrm{m} ; 200 \mathrm{keV} \mathrm{H}^{+}$} \\
\hline S.P. total & \multicolumn{3}{c}{$1.58 \times 10^{-12}$} & \multicolumn{4}{c}{$1.71 \times 10^{-13}$} \\
S.P. elastic & \multicolumn{3}{c}{$4.30 \times 10^{-13}$} & \multicolumn{3}{c}{$1.31 \times 10^{-16}$} \\
S.P. inelastic & \multicolumn{3}{c}{$1.15 \times 10^{-12}$} & \multicolumn{4}{c}{$1.71 \times 10^{-13}$} \\
\hline fl. & $D$ tot. & $D$ el. & $D$ inlel. & $f l$. & $D$ tot. & $D$ el. & $D$ inel. \\
\hline $6.3 \times 10^{12}$ & 1.5 & 0.4 & 1.1 & $6.3 \times 10^{13}$ & 1.6 & 0.001 & 1.6 \\
$3.8 \times 10^{13}$ & 9.1 & 2.5 & 6.6 & $2.5 \times 10^{14}$ & 6.6 & 0.005 & 6.6 \\
$7.5 \times 10^{13}$ & 18.3 & 5.0 & 13.3 & $2.0 \times 10^{15}$ & 52.7 & 0.040 & 52.7 \\
$1.5 \times 10^{14}$ & 36.5 & 9.9 & 26.6 & $4.0 \times 10^{15}$ & 105.5 & 0.080 & 105.4 \\
$6.0 \times 10^{14}$ & 146.0 & 39.7 & 106.3 & $8.0 \times 10^{15}$ & 211.0 & 0.161 & 210.8 \\
$5.2 \times 10^{15}$ & 1274.8 & 346.8 & 928.0 & $1.6 \times 10^{16}$ & 422.0 & 0.321 & 421.6 \\
\hline
\end{tabular}

Notes. S.P. - stopping power $\left(\mathrm{eV} \mathrm{cm}^{2} \mathrm{~mol}^{-1}\right), f l$. - fluence (ions $\left./ \mathrm{cm}^{2}\right), D-\operatorname{dose}(\mathrm{eV} /(16 \mathrm{u}))$.

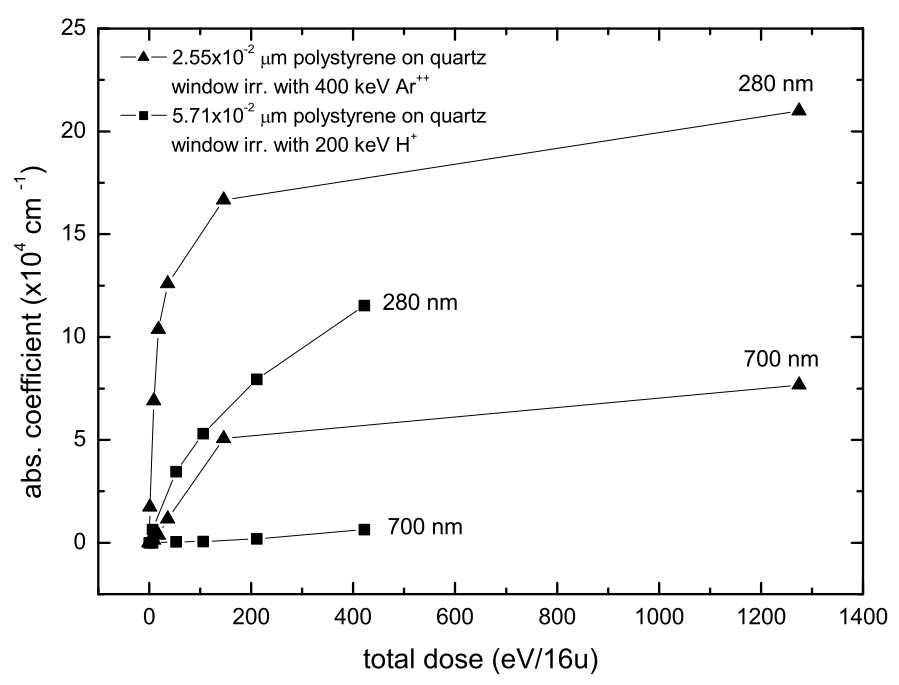

Fig. 6. Absorption coefficient of two polystyrene samples irradiated with protons and argon ions at two different wavelengths.

experiments was estimated with the SRIM (Zeigler et al. 1996) software, taking into account the thickness of the polystyrene layers used in the experiments $\left(\sim 10^{-2} \mu \mathrm{m}\right)$ and listed in Table 1.

Concerning the change of absorption coefficient with the ion fluence (i.e., with the energy absorbed by the polystyrene layer, see Fig. 4) it is clear that the absorption coefficient in the near UV region has a similar increasing trend for both ions $(\mathrm{H}$ and Ar), although the effect is greater for the heavier Ar. In the visible the difference between the two ions is more relevant. In Fig. 6 the absorption coefficient at two different wavelengths is plotted versus the energy released by ions through total (elastic plus inelastic) collisions between ions and target atoms to investigate the physical mechanism responsible for the modifications of the spectra. The amount of elastic/inelastic energy loss is a function of ion mass and energy and slightly depends on the target material. The energy lost by colliding ions is redistributed among the species present in the target, and three main effects occur: 1 . displacements of atoms from their lattice position; 2. ionization processes; and 3. creation of phonons. With the SRIM (Zeigler et al. 1996) software, it was computed that for $200 \mathrm{keV} \mathrm{H}^{+}$ions colliding with a polystyrene layer at the angle of $45^{\circ}$, almost all (99.9\%) energy is released by inelastic collisions. For $400 \mathrm{keV}$ $\mathrm{Ar}^{++}$, the fraction of energy released by inelastic collisions is less, $72.8 \%$. The stopping power of elastic collisions of lighter
$\mathrm{H}^{+}$ions $\left(1.31 \times 10^{-16} \mathrm{eV} \mathrm{cm}^{2} \mathrm{~mol}^{-1}\right)$ is much lower than that of heavier Ar ions $\left(4.30 \times 10^{-13} \mathrm{eV} \mathrm{cm}^{2} \mathrm{~mol}^{-1}\right)$ (see Table 1$)$.

Altough it has been shown that the changes induced by irradiation depend on the energy deposition process, a detailed description of the relevance of the different energy deposition mechanisms (elastic versus inelastic) in the modification processes is still lacking (Puglisi et al. 1986; Fink et al. 1994). According to our results, comparing the effect of Ar and $\mathrm{H}$ irradiation on the absorption coefficient it seems that the spectral changes of this organic material are mainly due to elastic collisions.

\subsection{Irradiation of organics deposited on silicates}

The next step in our experiments was the proton/argon irradiation of polystyrene layers deposited on olivine pellets.

The thickness of each polystyrene layer deposited on silicates was estimated on the base of the comparison of the peak area of an absorption feature at $\lambda=1680 \mathrm{~nm}$ seen in the reflectance spectrum of the pellet with the peak area of the same feature seen in transmittance on a polystyrene film of known thickness. For the computation of the expected reflectance $R^{*}$ of the irradiated polystyrene layer deposited on the silicate pellets, a simple model described by the following formulas was used

$$
\begin{aligned}
R^{*} & =R T^{2} \\
T & =\mathrm{e}^{-\tau},
\end{aligned}
$$

where $R$ is the reflectance of the silicate, $T$ is the transmittance of deposited polystyrene. The optical depth of an absorption feature $\tau$ measured in transmittance is

$$
\tau_{1}=\alpha s_{1}
$$

while when measured in reflectance it can be roughly estimated as

$\tau_{2}=2 \alpha s_{2}$

where $\alpha$ is the absorption coefficient and $s$ is the thickness of absorbing material. The final formula used for the computation of the thickness of deposited polystyrene layer is

$s_{2}=\frac{1}{2} \frac{A_{2}}{A_{1}} \cdot s_{1}$

where $A$ is the measured peak area obtained by integrating the optical depth over the band. In all equations the index 1 denotes the polystyrene film of known thickness $(50 \mu \mathrm{m})$, index 2 

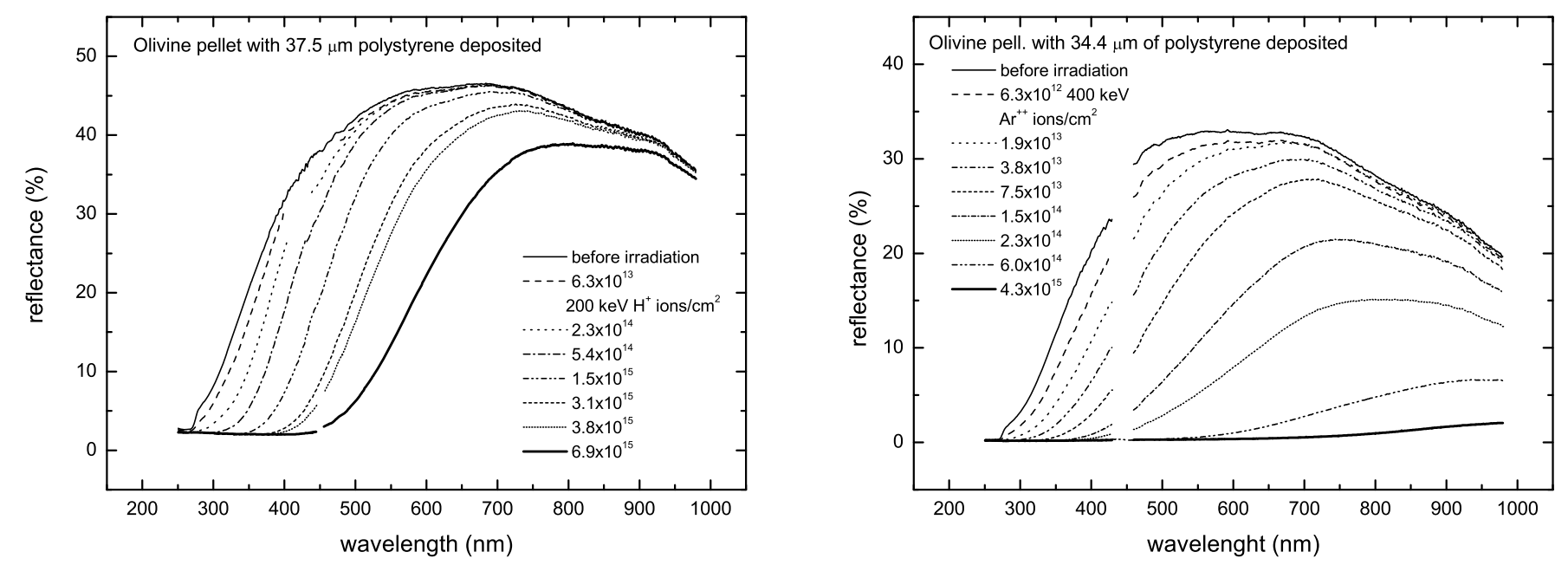

Fig. 7. Reflectance spectra of olivine pellets covered with a layer of polystyrene measured "in situ" during the irradiation.

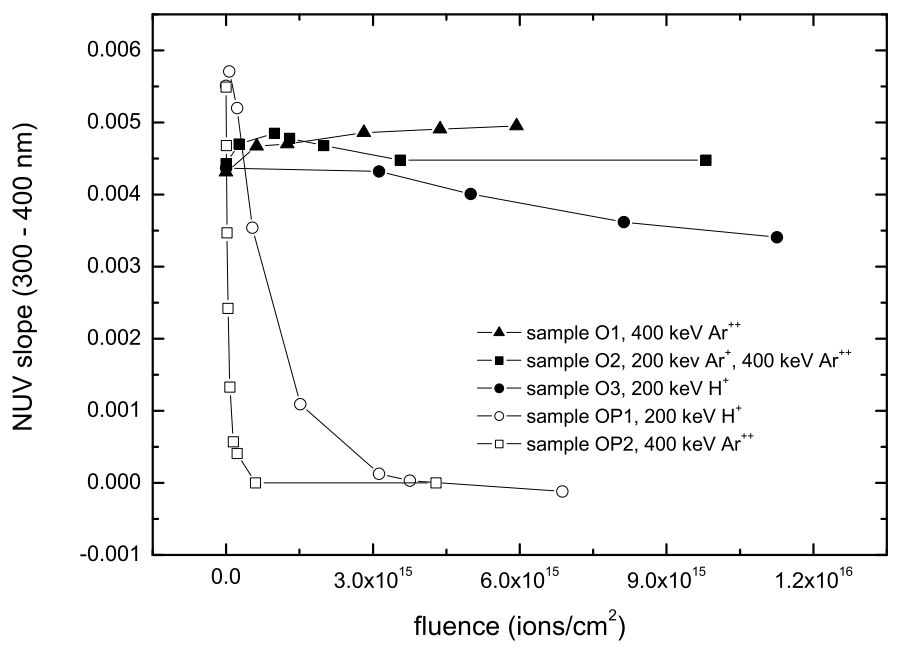

Fig. 8. Dependence of NUV spectral slopes of the samples on different ion fluence.

the polystyrene layer deposited on silicate. The thickness of polystyrene layer deposited on the olivine was estimated to be $37.50 \mu \mathrm{m}$ (sample OP1) and $34.43 \mu \mathrm{m}$ (sample OP2). Because both films are much ticker than the penetration depth of the ions used to irradiate them $\left(200 \mathrm{keV} \mathrm{H}^{+}\right.$and $\left.400 \mathrm{keV} \mathrm{Ar}^{++}\right)$, the spectral changes of the samples after irradiation can be attributed just to the chemical and physical changes of the irradiated polystyrene film, as the surface of the silicates cannot be affected by the incoming ions. With this condition we mean to demonstrate the effect of weathered organic matter, if present, on the spectra of any substrate.

Irradiation of sample OP1 with $200 \mathrm{keV} \mathrm{H}^{+}$and sample OP2 with $400 \mathrm{keV} \mathrm{Ar}^{++}$ions was done at room temperature with the apparatus described above. Reflectance spectra of both samples before, during, and after irradiation are shown in Fig. 7. The spectral slopes in NUV wavelength region were determined (see Fig. 8).

\section{Discussion}

It has been shown several times that amorphous carbon formation in ion irradiated carbon containing solid targets (both frozen and refractory) is a very general process (Baratta et al. 2004).
During ion-beam irradiation of different carbonaceous materials (characterized e.g. by different degree of order and/or different $\mathrm{sp}^{2} / \mathrm{sp}^{3}$ ratio), two competitive processes must be considered: graphitization and amorphization. The first one can be related to the energy release in form of heat ("thermal spikes") inside the collision cascade $\left(\mathrm{sp}^{3}\right.$ to $\mathrm{sp}^{2}$ conversion with or without $\mathrm{H}$ loss), while amorphisation is caused by the displacements due to collisions and depends on the size of the crystallites. At higher fluence the number of $\mathrm{sp}^{2}$ to $\mathrm{sp}^{3}$ conversions (declustering) balances the number of $\mathrm{sp}^{3}$ to $\mathrm{sp}^{2}$ conversion (graphitisation), the $\mathrm{sp}^{2} / \mathrm{sp}^{3}$ ratio does not increase any more - the saturation is reached (Compagnini et al. 1992). In the work of Baratta et al. (Baratta et al. 2004) it was shown that at saturation different ion-irradiated carbonaceous materials get a similar structure (Raman spectra, $\mathrm{H} / \mathrm{C}$ ratio, optical gap etc.) almost independently of the starting material even if the efficiency of the process depends on the initial chemical composition (see Ferini et al. 2004, and references therein). In this work we observed the decrease of the optical gap of polystyrene, and its value at the end of argon irradiation $(0.5 \mathrm{eV})$ demonstrates that the saturation occured (compare Compagnini et al. 1992). Hence polystyrene can be considered as a template that should describe from a qualitative point of view the darkening suffered by a surface covered with an organic layer exposed to cosmic ion irradiation, even if the darkening efficiency (time scale) varies with the exact composition of the layer.

As is described in the previous section, we used the reflectance spectra of irradiated polystyrene deposited over olivine samples to define the corrective factor used to compute the absorption coefficients at different fluences. In particular, we used the simple model expressed by Eq. (3) (and (1), (4)) where $R$ is the reflectance of the sample before irradiation, $T$ is the transmittance of irradiated polystyrene, $\alpha$ the absorption coefficient and $s$ the thickness of absorbing material.

We obtained the best fit of the measured and theoretical reflectance of the sample by changing the expected value of the irradiated (absorbing) depth of the polystyrene layer and the corrective factor of the continuum level used to derive the absorption coefficient from the transmittance spectra for each irradiation fluence. When the transmittance spectrum of polystyrene over quartz was not available at comparable fluences, we obtained a synthetic absorption coefficient by interpolation of two transmittance spectra of lower and higher fluence. As an example, the reflectance spectra of polystyrene over olivine before 

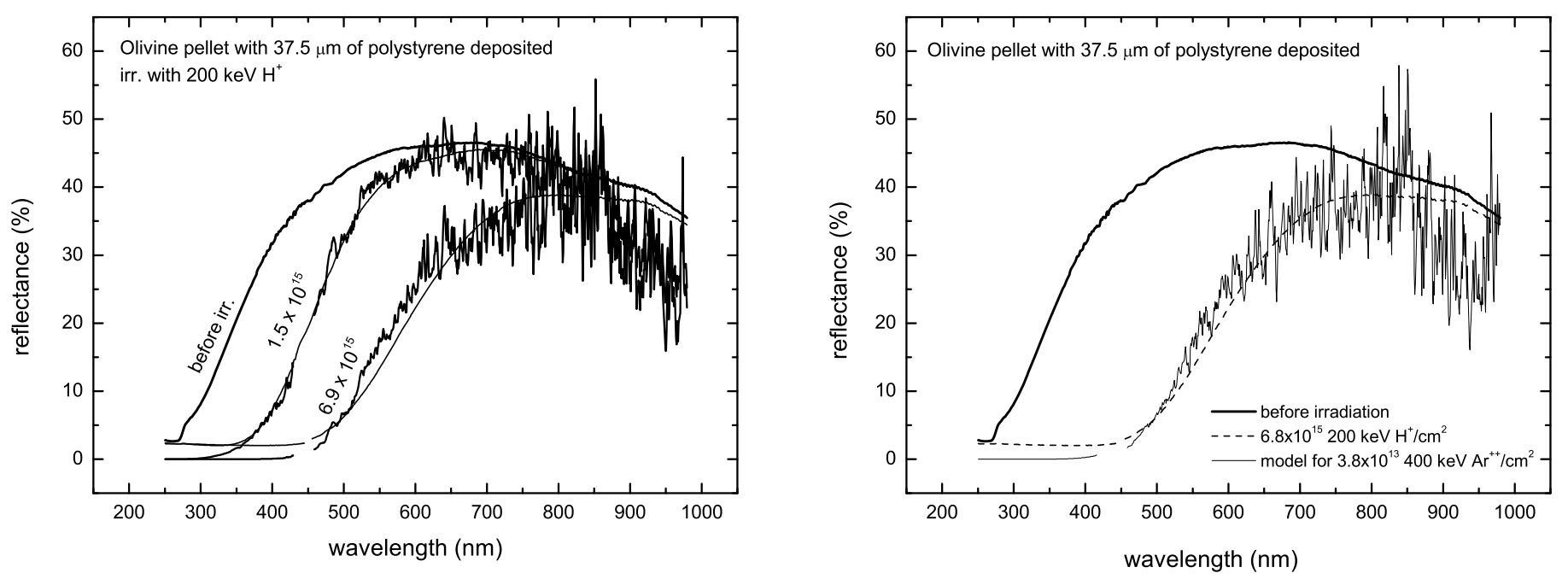

Fig. 9. Comparison between measured and expected reflectance of olivines with polystyrene layer deposited after $200 \mathrm{keV} \mathrm{H}^{+}$irradiation. Absorption coefficient of polystyrene after proton (left) and argon ions (right) irradiation was used to simulate the effect expected after the absorption of certain amount of energy deposited by ions.

and after irradiation at different fluences together with the corresponding best-fit spectra are reported in Fig. 9. We notice that the model curve is very noisy at higher wavelengths. As described below, this is due to the small values of the absorption coefficient in this spectral range (see Fig. 4).

In the geometrical configuration used for the reflectance measurements, the scattered light is collected by the spectrometer at an angle of $45^{\circ}$ with respect to the incident radiation (see Fig. 1), then the optical path is greater than the geometrical thickness. By using Eq. (2), the geometrical thicknesses, corresponding to the effective optical paths determined with the fitting procedure, were within the interval $1.0-1.7 \mu \mathrm{m}$. These values agree well with the penetration depth of $200 \mathrm{keV}$ protons into polystyrene computed by SRIM calculation, that is less than $2 \mu \mathrm{m}$. This is an order of magnitude lower than the thickness of polystyrene layer deposited on the olivine sample that is $37.50 \mu \mathrm{m}$.

The absorption coefficient of polystyrene after $\mathrm{H}^{+}$irradiation at the longer wavelengths is very low (Fig. 4), and due to the thin polystyrene layer used for its computation, it is determined with a high level of noise. The irradiated polystyrene layer deposited on the olivine pellet is two orders of magnitude thicker than the polystyrene layer deposited on the quartz window. The higher value of $s$ in Eqs. (1), (3) and (4) causes a corresponding high level of noise in the modeled reflectance - see Fig. 9.

Analogously by using Eqs. (3), (1), and (4) we simulated the effects of proton irradiation on sample OP1 using the absorption coefficient of polystyrene $(\alpha)$ after $400 \mathrm{keV} \mathrm{Ar}^{++}$irradiation (see right panel of Fig. 9). We found that it is possible to simulate the spectral changes of the sample after irradiation with $6.9 \times 10^{15} 200 \mathrm{keV}$ protons $/ \mathrm{cm}^{2}$ using the absorption coefficient of polystyrene irradiated with Ar ions at a fluence of $3.8 \times 10^{13}$ ions $/ \mathrm{cm}^{2}$. In this case, the best-fit thickness of irradiated material is $0.9 \mu \mathrm{m}$.

An analogous procedure was used to analyze the effects of $400 \mathrm{keV} \mathrm{Ar}^{++}$irradiation on sample OP2. By using the absorption coefficient of Ar irradiated polystyrene, the best fit at different fluences was obtained by determining the corrective factors of the transmittance spectra used to derive the absorption coefficients and for geometrical thickness ranging in the interval 0.2$0.3 \mu \mathrm{m}$ of irradiated polystyrene layer, which is significantly less than the penetration depth of argon ions (i.e. $0.4 \mu \mathrm{m}$ ) calculated by SRIM (Zeigler et al. 1996). All the corrective factors of the transmittance spectra derived with the best-fit procedure were well within $5 \%$ for both $200 \mathrm{keV}$ protons and $400 \mathrm{keV}$ Ar ions irradiations. This makes us confident of the reliability of the derived absorption coefficients of irradiated polystyrene. Anyway it should be considered that the computed absorption coefficients are not absolute, but must be considered as effective, i.e. they are able to describe the transmittance and reflectance spectra of irradiated polystyrene trough the rough optical transfer model here considered. In order to use more complex optical models that take into account for example surface roughness and volume scattering, the derivation of optical constants of the irradiated polystyrene film would be necessary.

In the left panel of Fig. 10 are plotted six spectra of irradiated sample OP2 at different ion fluences together with the bestfit theroretical spectra. Like for the proton irradiation of sample OP1, it was found that it is possible to simulate the argon irradiation effects on reflectance spectra with the absorption coefficient of polystyrene after proton irradiation (see the left panel of Fig. 10). In this case, the thickness of irradiated polystyrene was found to be $0.3-0.4 \mu \mathrm{m}$.

We note that for both ions the changes in the reflectance spectrum of the irradiated samples are only due to the external layers, as it usually occurs in cosmic ions irradiating Solar System objects.

In the experiment of Zhu et al. (2000) it was shown that due to the carbonization of material the absorption edge of polystyrene gradually shifts from the UV toward the visible with increasing ion fluences in the electronic energy loss regime (Compagnini et al. 1990); on the other hand, no significant changes could be observed in the near-infrared range. These findings agree with the $200 \mathrm{keV}$ proton irradiation case, where no significantly absorption was observed even at the highest irradiation fluence $\left(1.6 \times 10^{16} / \mathrm{cm}^{2}\right)$ in the polystyrene film above $900 \mathrm{~nm}$. On the other hand, we found that Ar ions significantly induce a darkening even in the NIR above $900 \mathrm{~nm}$. This different behavior could be related to the difference in the energy deposition process, because Ar ions significantly interact also through elastic collisions, while $200 \mathrm{keV}$ protons only interact through electronic excitations. Nevertheless, further experiments are necessary to confirm this hypothesis. 

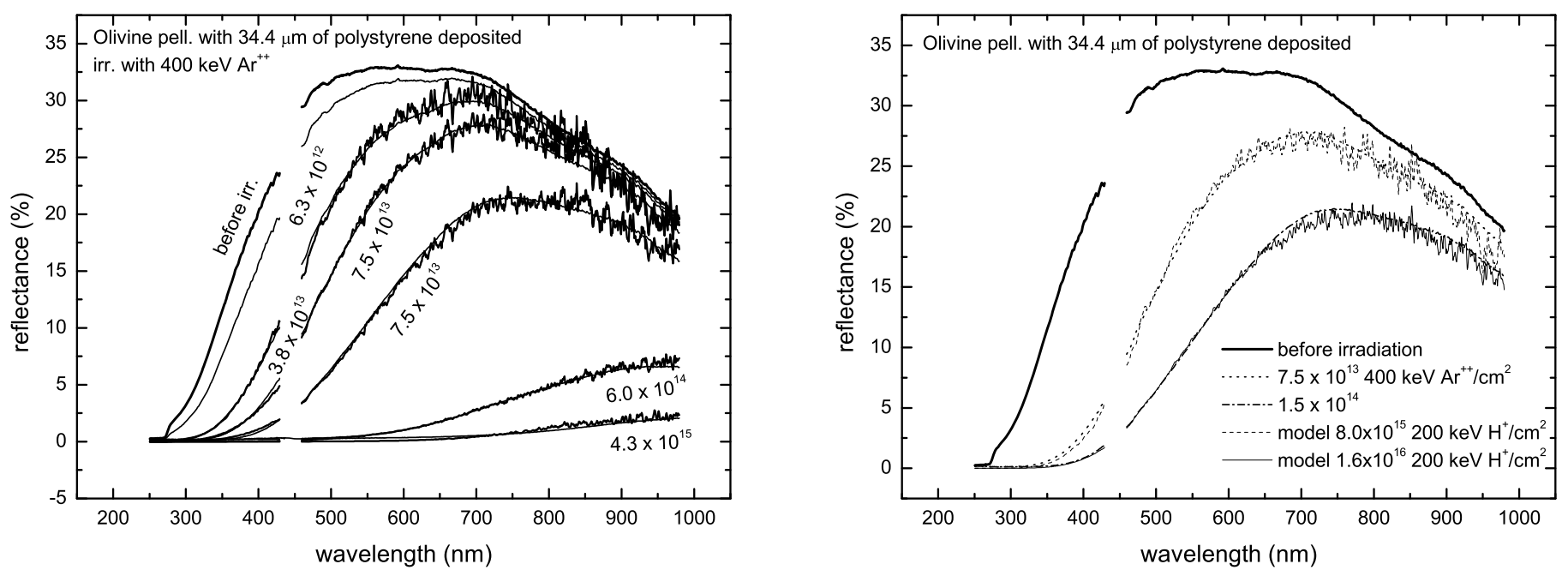

Fig. 10. Comparison between measured and expected reflectance of olivines with a polystyrene layer deposited after $400 \mathrm{kev}^{\mathrm{Ar}} \mathrm{r}^{++}$irradiation. The absorption coefficient of polystyren after argon ions (left) and protons (right) irradiation was used to simulate the effect expected after the absorption of certain amount of energy deposited by ions.

\subsection{Timescale and astrophysical applications}

There are a number of energetic ion populations bombarding the surfaces of small bodies in the outer Solar System: solar wind ions, ions from solar flares, galactic cosmic rays, and the so called "anomalous" cosmic ray component. The last component is relevant only to objects at the Pluto orbit and beyond though.

Solar wind is an expanding flux of fully ionized plasma that reaches at distances greater than a few solar radii an expansion speed about $400 \mathrm{~km} \mathrm{~s}^{-1}$ corresponding to an energy of $\approx 1 \mathrm{keV} / \mathrm{u}$ (slow solar wind, see e.g. Gosling 2007). Because the changes in the reflectance spectra of the irradiated olivine samples with polystyrene deposited are only due to the external layers, below we study the effect of the solar wind ions irradiation because their typical penetration depth is small - around $0.1 \mu \mathrm{m}-$ and they are the most abundant ions in the Solar System.

The expected effect of the solar wind ions on the sample of OP2 at different doses was simulated with the simple optical model described in Sect. 3.3. Ions of $1 \mathrm{keV} / \mathrm{u}$ deposit the energy into the polystyrene through both elastic and inelastic interactions, thus the absorption coefficient of polystyrene after $400 \mathrm{keV}$ Ar irradiation was considered in the model.

The corresponding timescale for the solar wind ions effect on the objects at the $2.7 \mathrm{AU}$ (the average heliocentric distance of the Main Belt of asteroids) was estimated by dividing the dose transferred into the material through the ions by the stopping power of considered solar wind ions multiplied by the appropriate ion flux.

We considered two cases: $1 \mathrm{keV} \mathrm{H}^{+}$i.e. the most abundant of the solar wind ions, and $36 \mathrm{keV} \mathrm{Ar}$. Here the $36 \mathrm{keV} \mathrm{Ar}^{+}$is considered as representative of all of the heavy ions. In particular, its flux is multiplied by a factor of 1000 to have an elastic dose corresponding to the total dose provided by all other solar wind ions ( $\mathrm{H}, \mathrm{He}, \mathrm{C}, \mathrm{O}, \mathrm{N}$, etc.; see Vernazza et al. 2006). The flux of protons $\approx 10^{8} \mathrm{~s}^{-1} \mathrm{~cm}^{-2}$ and of Ar ions $4 \times 10^{2} \mathrm{~s}^{-1} \mathrm{~cm}^{-2}$ at $1 \mathrm{AU}$ was scaled to $2.7 \mathrm{AU}$ considering a variation as the inverse squared heliocentric distance.

Then the timescale was multiplied by a factor of 4 to take into account the ratio between the geometrical cross-section and the whole surface of a spherical body. Simulated reflectance spectra of the olivine with a polystyrene layer deposited and corresponding timescales for $1 \mathrm{keV} \mathrm{H}^{+}$and $36 \mathrm{keV} \mathrm{Ar}^{+}$irradiation are ploted in Fig. 11.

Comparing the reflectance spectra of the olivine sample (Fig. 3) with the spectra of the olivine sample covered with polystyrene (right panel of Fig. 7) at different $400 \mathrm{keV} \mathrm{Ar}^{++}$irradiation fluences it is evident that the diffuse reflectance spectra of the silicate covered with the polystyrene layer exhibits a much more significant variation than that of pure silicate due to the carbonization of the irradiated organic coating. By considering the NIR part of the spectra and the early irradiation steps, the weathering process is $10^{2}$ times more efficient for the polystyrene coating.

On the basis of our irradiation experiments of silicates and organic matter, we can say that the spectral slope in NUV region $(300-400 \mathrm{~nm})$ is decreasing with increasing doses absorbed by material. The blueing in the NUV is accompanied by a reddening in the Vis-NIR range.

Looking at Fig. 8 it is evident that the spectral effect of ion irradiation in the NUV region is much higher for the irradiation of olivine with polystyrene deposited than for pure silicates. The decreasing trend of NUV spectral slope, accompanied by a reddening in the Vis-NIR range, would be a good support to the Hendrix \& Vilas (2006) suggestion stating that asteroid surfaces that are spectrally redder in the VNIR are also spectrally bluer in the NUV. According to Hendrix \& Vilas (2006), the addition of nanophase iron to the regolith through the weathering process could produce this observed correlation. An alternative explanation is given by our results that shows how surfaces covered with organic matter could give at least from a qualitative point of view the same correlation as a consequence of cosmic ion irradiation. Furthermore, the presence of organics on the SS body surfaces can greatly increase the effect of cosmic ion irradiation-induced space weathering.

As an example of the possible effect on the reflectance spectra of the small Solar System bodies if some irradidiated organic matter is present, we consider the unusually red spectrum of the Centaur 5145 Pholus. We consider a simplified scenario, in which the whole surface of Pholus is made of silicates, olivine in our model, covered with an initial transparent organic layer, such as polystyrene. (In reality, the surface of Pholus is composed also of different ices, e.g. Cruikshank et al. 1998, but, because they are transparent in the studied spectral region, we can omit 

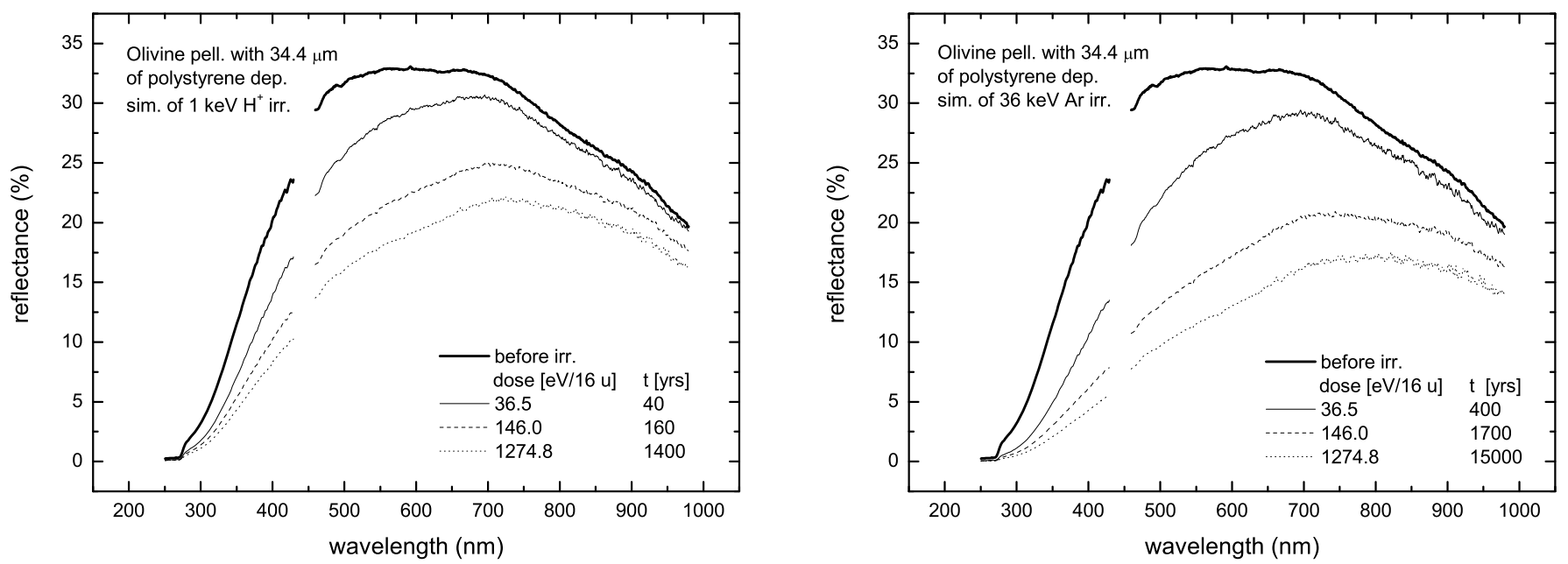

Fig. 11. Simulated reflectance spectra of olivine pellet with deposited polystyrene (sample OP2) irradiated with 1 keV hydrogen and $36 \mathrm{keV}$ argon ions.

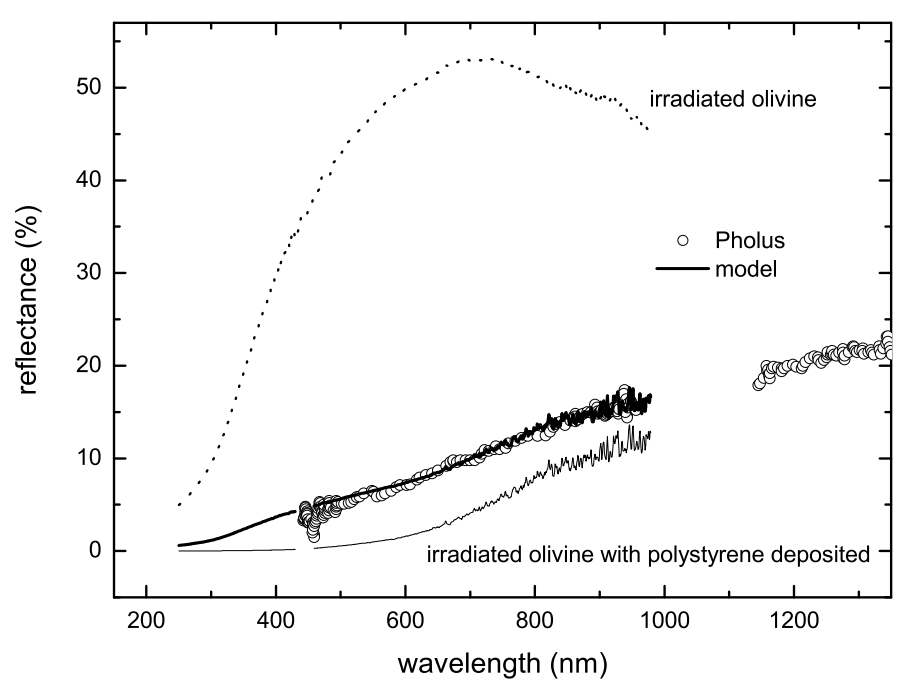

Fig. 12. Simulation of the reflectance spectra of 5145 Pholus. The spectral data of Pholus (Cruikshank et al. 1998) were fitted with a linear curve equal to $0.12 \times S_{\mathrm{o}}+0.88 \times S_{\mathrm{op}}$, where $S_{\mathrm{o}}$ is the reflectance spectra of irradiated olivine and $S_{\text {op }}$ is the reflectance spectra of irradiated olivine covered with a layer of polystyrene.

them in the model.) We allow in the model that a fraction of the silicate surface is not covered by the organic layer. Let us suppose that the organic layer has been exposed enough to be fully carbonized, i.e. enough to get the saturation. For polystyrene irradiated with $36 \mathrm{keV} \mathrm{Ar}^{+}$at $20 \mathrm{AU}$, that is roughly the semimajor orbital axis of Pholus, the saturation would be reached in $\approx 9 \times 10^{5}$ years. The uncovered olivine surface is weathered by the same ion fluence as well. Under these conditions, using the simple optical model here proposed, it is possible to simulate the observed spectrum of Pholus in UV-NIR part, as it is shown in Fig. 12. In the model the best fit was obtained for an organic covered surface fraction of $88 \%$ and a thickess of the irradiated organics of $0.18 \mu \mathrm{m}$, which is only twice the penetration depth of the solar wind ions. This implies that the estimated age of $9 \times 10^{5}$ years has to be considered as a lower limit.

\section{Summary}

With polystyrene as an example of organic (hydrocarbon) material it was shown that the effect of space weathering of Solar
System objects which are covered with some amount of organic material is more effective with respect to the objects composed of pure silicates. Moreover, it was shown that the space weathering effects could be so great for organic materials that if these are present on the asteroids, the space weathering effects on these objects is almost independent of the substrate. This claim is valid if the thickness of the organic layer is higher than the penetration depth of ions. If not, the irradiation effect on the substrate has to be taken into account. Particularly the spectral slope in the near UV spectral region decreases quickly at low doses, if organic matter is irradiated.

The ions used in this work $-200 \mathrm{keV} \mathrm{H}^{+}$and $400 \mathrm{keV} \mathrm{Ar}^{++}{ }_{-}$ could be a representation of two different kinds of ions inducing the space weathering of the Solar System objects in terms of the process of energy deposition into the material: $200 \mathrm{keV}$ protons as ions with only an inelastic contribution and $400 \mathrm{keV}$ argon with both inelastic and elastic components. It was shown that $\mathrm{Ar}$ ions induce spectral changes more efficiently than the protons, moreover, argon ions induce spectral darkening also in the NIR, while protons do not, not even at higher doses.

Measured absorption coefficients of irradiated polystyrene were successfully used to simulate the effect of ion irradiation of silicate covered with an organic layer. Moreover, the measured absorption coefficient of fully carbonized polystyrene was effectively used to simulate the UV-NIR part of the reflectance spectra of the Centaur 5145 Pholus. The fit of the unusually red spectra of Pholus was reached under the assumption of the relatively short exposition of its surface to the solar wind ion irradiation $\left(\approx 9 \times 10^{5}\right.$ years $)$.

The spectral changes in organic material induced by fast ions seem to be mainly due to the elastic part of the ion energy deposition into the material. However, more experiments are necessary, particularly with ions with a different ratio of elastic/inelastic energy deposition into the material to better understand the relevance of the different energy deposition mechanisms.

The data concerning the absorption coefficient of polystyrene are available on our website (http://web. ct.astro.it/weblab/dbindex.html) and can be used to compare laboratory with astronomical spectra. This will allow the community to obtain information on the amount of organics that contaminate the surface of a given object, as well as on the relevance of the exposure to cosmic ions (space weathering). 
Acknowledgements. This research has been supported by Italian Space Agency contract No. I/015/07/0 (Studi di Esplorazione del Sistema Solare). We thank F. Spinella for his help in the laboratory and M. E. Palumbo for her useful comments.

\section{References}

Baratta, G. A., Manella, V., Brucato J. R., et al. 2004, J. Raman Spec., 35, 487 Brunetto, R., \& Strazzulla, G. 2005, Icarus, 179, 265

Brunetto, R., Barucci, M. A., Dotto, E., \& Strazulla, G. 2006a, AJ, 644, 646

Brunetto, R., Romano, F., Blanco, A., et al. 2006b, Icarus, 180, 546

Chapman, C. R. 1996, Meteorit. Planet. Sci., 31, 699

Compagnini, G., Foti, G., Reitano, R., \& Mondio, G. 1990, Phys. Rev. Lett., 57, 2546

Compagnini, G., Calgano, L., \& Foti, G. 1992, Phys. Rev. Lett., 69, 454

Cruikshank, D. P., Roush, T. L., Bartholomew, M. J., et al. 1998, Icarus, 135, 389

Curró, G., Mondio, G., Neri, F., Foti, G., \& Compagnini, G. 1995, J. Elect. Spec. Rel. Phen., 132, 313

Ferini, G., Baratta, G. A., \& Palumbo M. E. 2004, A\&A, 414, 757

Fink, D., Hosoi, F., Omichi, H., Sasuga, T., \& Amaral, L. 1994, Radiat. Eff., 132, 313

Gosling, J. T. 2007, in Encyclopedia of the Solar System, second edn. (Academic Press), ed. L. McFadden, P. R. Weissman, \& T. V. Johnson, 99116

Hapke, B. 2001, J. Geophys. Res., 106, 10, 039
Hendrix, V., \& Vilas, F. 2006, ApJ, 132, 1396

Loeffler, M. J., Baragiola, R. A., \& Murayama, M. 2008, Icarus, 196, 285

Loeffler, M. J., Dukes, C. A., \& Baragiola, R. A. 2009, J. Geophys. Res., 114, E03003

Moroz, L., Baratta, G., Strazzulla, G., et al. 2004, Icarus, 170, 214

Palumbo, M. E., Baratta, G. A., Fulvio, D., et al. 2008, J. Phys., 101, 1

Partridge, R. 1967, J. Chem. Phys., 47, 4223

Pieters, C. M., Fischer, E. M., Rode, O., \& Basu, A. 1993, J. Geophys. Res., 98, 20817

Pieters, C. M., Taylor, L. A., Noble, S. K., et al. 2000, Meteorit. Planet. Sci., 35, 1101

Puglisi, O., Licciardello, S., Pignataro, L., Calgagno, \& Forti, G. 1986, Radiat. Eff., 98, 161

Sasaki, S., Nakamura, K., Hamabe, Y., Kurahashi, E., \& Hiroi, T. 2001, Nature, 410,555

Shkuratov, Yu. G., Stunkevich, N. P., \& Antipova-Karataeva, I. I. 1987, in Abstracts of the Lunar and Planetary Science Conference, 18, 555

Strazzulla, G., Dotto, E., Binzel, R., et al. 2005, Icarus, 174, 31

Tauc, J., Grigorovici, R., \& Vancu, A. 1966, Phys. Status Solidi, 15, 627

Vernazza, P., Brunetto, R., Strazzulla, G., et al. 2006, A\&A, 451, L43

Wittmershaus, B. P., Baseler, T. T., Beaumont, G. T., \& Zhang, Y. 2002, J. Luminescence, 96, 107

Zeigler, J. F., Biersack, J. P. \& Littmark, U. 1996, The stopping and Range of Ions in Solids (New York: Pergamon Press)

Zhu, Z., Jin, Y., Liu, C., et al. 2000, Nuclear Instr. Met. Phys. Res. B., 166, 621 